\title{
An Extension of TODIM with VIKOR approach based on Gini Simpson Index of Diversity under Picture fuzzy framework to Evaluate Opinion Polls
}

\author{
Sunit Kumar ${ }^{\mathrm{a}}$, Satish Kumar ${ }^{\text {b }}$ \\ ${ }^{a}$ Department of Mathematics, Maharishi Markandeshwar (Deemed to be University), Mullana, Haryana, India \\ ${ }^{B}$ Department of Mathematics, Maharishi Markandeshwar (Deemed to be University), Mullana, Haryana, India \\ Email: ${ }^{\mathrm{A}}$ sunitgoel2009@gmail.com, ${ }^{\mathrm{B}}$ drsatish74@ rediffmail.com
}

Article History: Received: 10 November 2020; Revised 12 January 2021 Accepted: 27 January 2021; Published online: 5 April 2021

\begin{abstract}
Cuong and Kreinovich was the first who gives the idea of Picture fuzzy set (PFS), which is an extension of intuitionistic fuzzy set (IFS) by cosidering positive, negative and neutral membership of element. In this paper, we have been worked on new entropy measure of PFS from the probabilistic view point and it's properties are examined from mathematical point of view. A hybrid aproach is presented with the assistance of TODIM (Portuguese abbreviation for Interactive MultiCriteria Decision Making) and VIKOR (Vlsekriterijumska Optimizacija I Kompromisno Resenje) methods. Further, we applied it to MCDM (multi criterion decision making) problems with picture fuzzy numbers (PFNs), where the information about criteria synthetic weights is partially known and completely unknown and show its existence with the help of some practical cases. After getting the output, we are able to infer that the proposed hybrid approach is comparatively better so as to handle the uncertainty and vulnerabilities for the decision making problems. Based upon these two approaches we can determine the opinion poll of voting outcomes and then, we compare its result with other MCDM approaches that exists in the literature.
\end{abstract}

Keywords: Picture fuzzy sets, Distance measure, Picture fuzzy numbers, Hybrid TODIM-VIKOR.

MS Classification: 94A15, 94A24, 26D15

\section{Introduction}

Zadeh (1965) was the one who developed the basic idea of Fuzzy set (FS) which plays a significant role in decision making under uncertainty which contains only a membership degree. Various theories has come up to measure the uncertainity like probability theory, Zadeh's FS theory [1,2,20,27], IFS theory [3], hesitant fuzzy set theory [4, 5], rough set theory [6]. A significant generalization of FS is IFS which was proposed by Atanassov [3] has recieved much attention. The concept of IFS theory is that it assigns a membership degree ,non membership degree from 0 to 1 to every element the sum of membership $(\rho)$ and non-membership $(\mu)$ cannot exceed one. Various authors used the IFS in various fields from the application point of view. Atanassov [3] introduced the third element $(v)$ that satisfies $\rho+\mu+v=1$ where $v$ is known as "intuitionistic index". Neutral membership has not been considered in IFS. We found the concept of neutral degree in various situations when we go through the human opinion like: sure, refrain, no, denial. In this case we can not use IFS. To overcome this type of problems Cuong [8] proposed picture FS which is generalization of (FS) [1, 9] and (IFS)[3] with the active introduction of the positive $(\rho)$, neutral $(\mu)$, negative $(\eta)$ and refusal membership degree $(\phi)$, respectively.

Various researchers like, Wei [5] suggested some process to measure similarity between PFS, a voting method which support two-tuple linguistic PF preference relation proposed by Nie and co-authors [10], Peng and Dai [11] put forwarded an algorithm for picture fuzzy (PF) framework supported new distance measure and applied it to the decision making problems, Son [12] extended basic picture distance for PFSs clustering while representing the benefities and reasons of using PFSs. There are several studies which have been used in the MCDM methods with picture fuzzy information [5, 13, 15-17]. However, such a lot of work has been done by many researchers on picture FSs, but to the best of authors expertise, a very less research is being done on the picture fuzzy entropy from probabilistic view-point.

In multi criteria decision making problems, we intend to find the best possible alternative from a finite set of alternatives satisfying an explicit set of criteria. Sometimes it is very difficult to find that which mobile or which TV we have to purchase these are some real time examples of MCDM problems. Opricovic [26] was the first who proposed VIKOR method which is widely used in solving MCDM problems and it is an effective tool to measure the compromised solution. 
Hwang and Yoon [18] proposed TODIM method which is one of the most effective tool used to deal with decision making problems under uncertainty and risk especially in economics, medical sciences, social sciences, engineering etc. Many authors have worked on TODIM approaches [6,7,10, 19, 22-25]. But until now, no focus is given on hybrid TODIM-VIKOR methods for PFNs, which is based on entropy weight information.

The main motive of this paper is to combine the concept of TODIM and VIKOR approaches to tackle with the MCDM problems with PFNs. The main contribution are : (a).To introduce a new criteria for PF-entropy, (b). To contribute a new PF information measure which is based on Gini Simpson entropy, (c) To introduce an extended PF-TODIM-VIKOR method under picture fuzzy framework, which fully takes the advantags of entropy information, prospect theory and compromise solution, (d) A numerical example of election outcomes is described to verify the practical applicibility of the developed algorithm, (e) A deatiled comparative study and discussion are put forward to illustarte the superiority and effectiveness of the developed algorithm.

In the first section, we discuss the work done by many researchers in this field also the motivational source. Second section iden application to voting model by using the the proposed hybrid MCDM technique. In the last segment, the paper is presented with Conclusions .

\section{Preliminaries}

Some basic definitions and concepts related to FS, IFS and PFS over $Z^{*}=\left\{\xi_{1}, \xi_{2}, \ldots, \xi_{n}\right\}$ has been discussed in this section.

Definition 2.1 A FS E in $\mathrm{Z}^{*}$ is defined as [1]:

$$
E=\left\{\left(\xi_{i}, \rho_{E}\left(\xi_{i}\right)\right): \xi_{i} \in Z^{*}\right\},
$$

where $\rho_{E}: Y^{*} \rightarrow[0,1]$ is called membership function and $\rho_{E}\left(\xi_{i}\right) \in[0,1]$ called as membership degree of $Y^{*}$.

Definition 2.2 [3] An IFS E on $\mathrm{Y}^{*}$ is defined as:

$$
E=\left\{\left(\xi_{i}, \rho_{E}\left(\xi_{i}\right), v_{E}\left(\xi_{i}\right)\right): \xi_{i} \in Y^{*}\right\},
$$

where

$\rho_{E}: Y^{*} \rightarrow[0,1]$ and $v_{E}: Y^{*} \rightarrow[0,1]$, with $0 \leq \rho_{E}\left(\xi_{i}\right)+v_{E}\left(\xi_{i}\right) \leq 1$, for all $\xi_{i} \in Y^{*}$. For an IFS, the pair $\left(\rho_{E}\left(\xi_{i}\right), v_{E}\left(\xi_{i}\right)\right)$ is described as an IFN and denotes the membership and non membership degree of set E.

For each IFS $E$ in $Y^{*}$, the number $\phi_{E}\left(\xi_{i}\right)=1-\rho_{E}\left(\xi_{i}\right)-v_{E}\left(\xi_{i}\right), \xi_{i} \in Y^{*}$. Also, $\phi_{E}\left(\xi_{i}\right)$ shows the hesitancy degree. Obviously, when $\phi_{E}\left(\xi_{i}\right)=0$, that is $v_{E}\left(\xi_{i}\right)=1-\rho_{E}\left(\xi_{i}\right)$ for all $\xi_{i} \in Y^{*}$, IFS $E$ alters an ordinary FS.

There is a drawback in the IFS of Atanassov. In IFS, he has not defined the concept of 'degree of refusal' which restricts it's extent of application. This drawback was eliminated by Cuong and Kreinovich they added the 'degree of refusal membership' in Picture fuzzy set (PFS)'.

Definition 2.3 (Cuong BC2013) A picture FS E on set $\mathrm{Y}^{*}$ is defined as:

$$
E=\left\{\left(\xi_{i}, \rho_{E}\left(\xi_{i}\right), v_{E}\left(\xi_{i}\right), \eta_{E}\left(\xi_{i}\right)\right): \xi_{i} \in Y^{*}\right\}
$$

where

$$
\rho_{E}: Y^{*} \rightarrow[0,1], v_{E}: Y^{*} \rightarrow[0,1], \eta_{E}: Y^{*} \rightarrow[0,1],
$$

and $\rho_{E}\left(\xi_{i}\right), v_{E}\left(\xi_{i}\right), \eta_{E}\left(\xi_{i}\right)$ lies between 0 and 1 and shows the positive, neutral and non/negative membership degrees of set $E$ with the condition $0 \leq \rho_{E}\left(\xi_{i}\right)+v_{E}\left(\xi_{i}\right)+v_{E}\left(\xi_{i}\right) \leq 1$, for all $\xi_{i} \in Y^{*}$. Moreover, a degree of refusal membership $\phi_{E}\left(\xi_{i}\right)$ of $\xi_{i}$ in $E$ can be estimated accordingly as:

$$
\phi_{E}\left(\xi_{i}\right)=1-\rho_{E}\left(\xi_{i}\right)-v_{E}\left(\xi_{i}\right)-\eta_{E}\left(\xi_{i}\right)
$$

Obviously, when $v_{E}\left(\xi_{i}\right)=0$, then the PFSs reduce into IFS, while if $v_{E}\left(\xi_{i}\right), \eta_{E}\left(\xi_{i}\right)=0$ then the PFSs become FSs. In the voting, those who are abstain can be interpreted as: on one hand, they vote for; on the other hand, they vote against. Meanwhile, those who are refusal of the voting can be explained as they are not care about this voting.

For convenience, the pair $E=\left(\rho_{E}\left(\xi_{i}\right), v_{E}\left(\xi_{i}\right), \eta_{E}\left(\xi_{i}\right), \phi_{E}\left(\xi_{i}\right)\right)$ is called a PFN and every PFN is denoted by $\gamma=\left(\rho_{\gamma}, v_{\gamma}, \eta_{\gamma}, \phi_{\gamma}\right)$, where $\rho_{\gamma} \in[0,1], v_{\gamma} \in[0,1], \eta_{\gamma} \in[0,1], v_{\gamma} \in[0,1], \phi_{\gamma} \in[0,1]$ and $\rho_{\gamma}+v_{\gamma}+\eta_{\gamma}+\phi_{\gamma}=1$. Sometimes, we omit $\phi_{\gamma}$ and in short, we denote a PFN as $\beta=\left(\rho_{\gamma}, v_{\gamma}, \eta_{\gamma}\right)$.

Definition 2.4 (Cuong [8],Son H[12]) Suppose two PFNs are $\gamma_{1}=\left(\rho_{\gamma_{1}}, v_{\gamma_{1}}, \eta_{\gamma_{1}}\right)$ and $\gamma_{2}=\left(\rho_{\gamma_{2}}, v_{\gamma_{2}}, \eta_{\gamma_{2}}\right)$ and the Hamming distance measures between them computed as follows: 


$$
d_{H}\left(\gamma_{1}, \gamma_{2}\right)=\frac{1}{3}\left[\left(\left|\rho_{\gamma_{1}}-\rho_{\gamma_{2}}\right|\right)+\left(\left|v_{\gamma_{1}}-v_{\gamma_{2}}\right|\right)+\left(\left|\eta_{\gamma_{1}}-\eta_{\gamma_{2}}\right|\right)\right]
$$

Definition 2.5 For every two PFSs E and F, Cuong et al. $[8,16]$ defined some operations in the universe $\mathrm{Y}^{*}$ as following.

1. $E \subseteq F$ iff $\forall \xi_{i} \in Z^{*}, \rho_{M}\left(\xi_{i}\right) \leq \rho_{N}\left(\xi_{i}\right), v_{M}\left(\xi_{i}\right) \leq v_{N}\left(\xi_{i}\right), \eta_{M}\left(\xi_{i}\right) \geq \eta_{N}\left(\xi_{i}\right)$;

2. $E=F$ iff $\forall \xi_{i} \in Z^{*}, E \subseteq F$ and $F \subseteq E$;

3. $E \cap F=\left\{\rho_{M}\left(\xi_{i}\right) \wedge \rho_{N}\left(\xi_{i}\right), v_{M}\left(\xi_{i}\right) \wedge \rho_{N}\left(\xi_{i}\right)\right.$, and $\left.\eta_{M}\left(\xi_{i}\right) \vee \eta_{N}\left(\xi_{i}\right) \mid \xi_{i} \in Y^{*}\right\}$;

4. $E \cup F=\left\{\rho_{M}\left(\xi_{i}\right) \vee \rho_{N}\left(\xi_{i}\right), v_{M}\left(\xi_{i}\right) \wedge \rho_{N}\left(\xi_{i}\right)\right.$, and $\left.\eta_{M}\left(\xi_{i}\right) \wedge \eta_{N}\left(\xi_{i}\right) \mid \xi_{i} \in Y^{*}\right\}$.

5. If $E \subseteq F$ and $F \subseteq P$ then $E \subseteq P$;

6. $\left(E^{c}\right)^{c}=E$;

7. $\operatorname{co} E=E^{c}=\left\{\left(\xi_{i}, \eta_{M}\left(\xi_{i}\right) v_{M}\left(\xi_{i}\right), \rho_{M}\left(\xi_{i}\right) \mid \xi_{i} \in Y^{*}\right)\right\}$.

We introduce the following comparison laws to compare the two PFNs.

Definition 2.6 Wang et al. [6] Let $\gamma_{1}=\left(\rho_{\gamma_{1}}, v_{\gamma_{1}}, \eta_{\gamma_{1}}\right)$ and $\gamma_{2}=\left(\rho_{\gamma_{2}}, v_{\gamma_{2}}, \eta_{\gamma_{2}}\right)$ be two PFNs. H( $\left.\gamma_{i}\right)$ be the accuracy degree and score $\left(\gamma_{i}\right)$ be the score function values then:

- If $\operatorname{score}\left(\gamma_{1}\right)<\operatorname{score}\left(\gamma_{2}\right)$, then $\gamma_{1}<\gamma_{2}$;

- If $\operatorname{score}\left(\gamma_{1}\right)=\operatorname{score}\left(\gamma_{2}\right)$, then

(a).If $H\left(\gamma_{1}\right)<H\left(\gamma_{2}\right)$, implies that $\gamma_{2}$ is superior to $\gamma_{1}$, denoted by $\gamma_{1}<\gamma_{2}$.

(b). If $H\left(\gamma_{1}\right)=H\left(\gamma_{2}\right)$, implies that $\gamma_{1}$ is equivalent to $\gamma_{2}$, denoted by $\gamma_{1} \equiv \gamma_{2}$;

Here $\operatorname{score}(\gamma)=\rho_{\gamma}-\eta_{\gamma}$ represents goal difference and $H(\gamma)=\rho_{\gamma}+v_{\gamma}+\eta_{\gamma}$ repesents an effective degree of voting. As score $\gamma$ increases, then the number of peoples are more who vote for' and who vote against' $\gamma$ and people who refuse of voting become less. So, $H(\gamma)$ demonstrates the effective degree of voting.

Definition 2.7 Wang et al. [6] introduced some laws for any PFNs $\gamma_{1}=\left(\rho_{\gamma_{1}}, v_{\gamma_{1}}, \eta_{\gamma_{1}}\right), \beta_{2}=\left(\rho_{\gamma_{2}}, v_{\gamma_{2}}, \eta_{\gamma_{2}}\right)$.

(1). $\gamma_{1} \otimes \gamma_{2}=\left(\rho_{\gamma_{1}}+v_{\gamma_{1}}\right)\left(\rho_{\gamma_{2}}+v_{\gamma_{2}}\right)-v_{\gamma_{1}} v_{\gamma_{2}}, v_{\gamma_{1}} v_{\gamma_{2}}, 1-\left(1-\eta_{\gamma_{1}}\right)\left(1-\eta_{\gamma_{2}}\right)$;

(2). $\gamma_{1}^{n}=\left(\rho_{\gamma_{1}}+v_{\gamma_{1}}\right)-v_{\gamma_{1}}^{n}, v_{\gamma_{1}}^{n} 1-\left(1-\eta_{\gamma_{1}}\right)^{n}$ for $n>0$.

\section{Entropy Concept for PFSs}

The fuzzy entropy measures the uncertainty of a FS and denote it's degree of fuzziness and to measure the fuzziness, the four axioms are proposed by De Luca and Termini [29] :

Definition 3.1 A function $E^{\wedge} \rightarrow[0, \infty)$ is called fuzzy entropy if $E^{\wedge}$ fulfills the folowing properties:

A1 (Sharpness):For all $E \in F S\left(Z^{*}\right), E^{\wedge}(E)=0$ iff set $E$ is crisp, i.e., $\mu_{E}=0.5$ for all $E \in F S\left(Z^{*}\right)$.

A2 (Maximality): The value of $\hat{E}(E)$ is maximum $\Leftrightarrow E$ is the most fuzzy set.

A3 (Resolution): $E^{\wedge}(E) \geq E^{\wedge}\left(E^{*}\right)$, where $E^{*}$ is the sharpened version .

A4 (Symmetry): $E^{\wedge}(E)=E^{\wedge}\left(E^{c}\right)$, where $E^{\wedge}\left(E^{c}\right)$ is the complement set of $E$.

Hung and Yang [14] introduced a new entropy measure for IFS which is defined as :

The function $\Theta: \operatorname{IFS}\left(\mathrm{Y}^{*}\right) \rightarrow[0, \infty)$ is said to be an entropy on IFS after satisifying the below said properties:

(I1) Sharpness: $\Theta(E)=0 \Leftrightarrow \Theta$ is a crisp set.

(I2) Maximality: $\Theta(E)=1$, will be maximum $\Leftrightarrow \rho_{E}\left(\xi_{i}\right)=v_{E}\left(\xi_{i}\right)=\phi_{E}\left(\xi_{i}\right)=\frac{1}{3}$, for all $\xi_{i} \in Y^{*}$

(I3) Symmetry: $\Theta(E)=\Theta\left(E^{c}\right)$, where $E^{c}$ is the complement of set $E$.

(I4) Resolution: $\Theta(E) \leq \Theta(F) \Leftrightarrow E \subseteq F$, i.e., $\rho_{E} \leq \rho_{F}$ and $v_{E} \leq v_{F}$ for $\max \left(\rho_{F}, v_{F}\right) \leq \frac{1}{3}$ and $\rho_{E} \geq \rho_{F}$ and $v_{E} \geq v_{F}$ for $\min \left(\rho_{F}, v_{E}\right) \geq \frac{1}{3}$. 
PFS is the further extension of IFS by adding one more component, that is $(\rho, v, \eta, \phi)$ satisfying the conditions $0 \leq \rho, v, \eta, \phi \leq 1$ and $\rho+v+\eta+\phi=1$.

Definition 3.3 A function en: $\operatorname{PFSs}\left(\mathrm{Y}^{*}\right) \rightarrow[0, \infty)$ is an entropy on PFS if en holds the subsequent four axiomatic requirements:

(W1) Sharpness:en $(E)=0 \Leftrightarrow E$ is a crisp set.

(W2) Maximality:en $(E)=1$, that is, attains maximum value $\Leftrightarrow \rho_{e n}\left(\xi_{i}\right)=v_{e n}\left(\xi_{i}\right)=\eta_{e n}\left(\xi_{i}\right)=\phi_{e n}\left(\xi_{i}\right)=$ $\frac{1}{4}$,for all $\xi_{i} \in Y^{*}$.

(W3) Symmetry:en $(E)=e n\left(E^{c}\right)$, where $E^{c}$ is the complement of $E$.

(W4) Resolution:en $(E) \leq e n(F)$ if $E$ is less fuzzy than $F$, that is $\rho_{E} \leq \rho_{F}, v_{E} \leq v_{F}$ and $\eta_{E} \leq \eta_{F}$ for max $\left(\rho_{F}, v_{F}, \eta_{F}\right) \leq \frac{1}{4}$ and $\rho_{E} \geq \rho_{F}, v_{E} \geq v_{F}$ and $\eta_{E} \geq \eta_{F}$ for $\min \left(\rho_{F}, v_{F}, \eta_{F}\right) \geq \frac{1}{4}$.

\section{Novel Parametric Measure for PFSs}

In this section, we proposed a new PF information measure based on Gini Simpson entropy.

\subsection{Background}

Let $\Delta_{n}=\left\{Y^{*}=\left(\xi_{1}, \xi_{2}, \ldots, \xi_{n}\right): \xi_{i} \geq 0, \sum_{i=1}^{n} \xi_{i}=1\right\}, n \geq 2$ be a finite set of complete probability distribution for some $Y^{*} \in \Delta_{n}$, the entropy given by

$$
V_{2}(Z)=\left[\sum_{i=1}^{n}\left(\xi_{i}^{\frac{1}{2}}-\xi_{i}^{2}\right]\right.
$$

Theorem 4.1 The entropy measure $\mathrm{V}_{2}(\mathrm{Z}), \mathrm{Z} \in \Delta_{\mathrm{n}}$,

the following properties has satisfied in the Gini Simpson fuzzy entropy [27]:

1. Symmetry: $V_{2}\left(\xi_{1}, \xi_{2}, \ldots, \xi_{n}\right)$ is a symmetric function of $\left(\xi_{1}, \xi_{2}, \ldots, \xi_{n}\right)$.

2. Non-Negative: $V_{2}(Z) \geq 0$.

3. Expansible: $V_{2}\left(\xi_{1}, \xi_{2}, \ldots, \xi_{n}, 0\right)=V_{2}\left(\xi_{1}, \xi_{2}, \ldots, \xi_{n}\right)$.

4. Decisive: $V_{2}(0,1)=0=V_{2}(1,0)$.

5. Maximility: $V_{2}\left(x_{1}, x_{2}, \ldots, x_{n}\right) \leq V_{2}\left(\frac{1}{n}, \frac{1}{n}, \ldots, \frac{1}{n}\right)$.

6. Concavity: $V_{2}\left(t S_{1}+(1-t) S_{2}\right) \geq V_{2}\left(S_{1}\right)+(1-t) V_{2}\left(S_{2}\right)$.

7. Continuity: $V_{2}\left(\xi_{1}, \xi_{2}, \ldots, \xi_{n}\right)$ is continuous for $\xi_{i} \geq 0(i=1,2, \ldots, n)$.

\subsection{Proposed PF Information Measure:}

For any $E \in P F S s$, we define

$$
\begin{aligned}
V_{P F S}(E)= & \frac{2}{3 n} \sum_{i=1}^{n}\left[\left(\rho_{E}\left(\xi_{i}\right)^{\frac{1}{2}}+v_{E}\left(\xi_{i}\right)^{\frac{1}{2}}+\eta_{E}\left(\xi_{i}\right)^{\frac{1}{2}}+\phi_{E}\left(\xi_{i}\right)^{\frac{1}{2}}\right)\right. \\
& \left.-\left(\rho_{E}\left(\xi_{i}\right)^{2}+v_{E}\left(\xi_{i}\right)^{2}+\eta_{E}\left(\xi_{i}\right)^{2}+\phi_{E}\left(\xi_{i}\right)^{2}\right)\right] .
\end{aligned}
$$

\section{Particular Cases:}

1. If $v_{E}\left(\xi_{i}\right)=0$ (nuteral membership), then PF entropy reduces to gini simpson IF entropy.

$$
\text { i.e. } \begin{aligned}
V_{2}(E)= & \frac{2}{3 n} \sum_{i=1}^{n}\left[\left(\rho_{E}\left(\xi_{i}\right)^{\frac{1}{2}}+\eta_{E}\left(\xi_{i}\right)^{\frac{1}{2}}+\phi_{E}\left(\xi_{i}\right)^{\frac{1}{2}}\right)\right. \\
& \left.-\left(\rho_{E}\left(\xi_{i}\right)^{2}+\eta_{E}\left(\xi_{i}\right)^{2}+\phi_{E}\left(\xi_{i}\right)^{2}\right)\right] .
\end{aligned}
$$

2. If $\eta_{E}\left(\xi_{i}\right)=0, \phi_{E}\left(\xi_{i}\right)=0$, then (4.5) reduces to the Gini Simpson fuzzy entropy [27] .

$$
V_{2}(E)=\frac{2}{3 n} \sum_{i=1}^{n}\left[\left(\rho_{E}\left(\xi_{i}\right)^{\frac{1}{2}}+\left(1-\rho_{E}\left(\xi_{i}\right)\right)^{\frac{1}{2}}-\left(\rho_{E}\left(\xi_{i}\right)^{2}+\left(1-\rho_{E}\left(\xi_{i}\right)^{2}\right] .\right.\right.\right.
$$

Proposition 4.1:

Under the condition $W 4$, we have 


$$
\begin{aligned}
& \left|\rho_{E}\left(\xi_{i}\right)-\frac{1}{4}\right|+\left|v_{E}\left(\xi_{i}\right)-\frac{1}{4}\right|+\left|\eta_{E}\left(\xi_{i}\right)-\frac{1}{4}\right|+\left|\phi_{E}\left(\xi_{i}\right)-\frac{1}{4}\right| \\
& \quad \geq\left|\rho_{F}\left(\xi_{i}\right)-\frac{1}{4}\right|+\left|v_{F}\left(\xi_{i}\right)-\frac{1}{4}\right|+\left|\eta_{F}\left(\xi_{i}\right)-\frac{1}{4}\right|+\left|\phi_{F}\left(\xi_{i}\right)-\frac{1}{4}\right|
\end{aligned}
$$

and

$$
\begin{aligned}
& {\left[\rho_{E}\left(\xi_{i}\right)-\frac{1}{4}\right]^{2}+\left[v_{E}\left(\xi_{i}\right)-\frac{1}{4}\right]^{2}+\left[\eta_{E}\left(\xi_{i}\right)-\frac{1}{4}\right]^{2}+\left[\phi_{E}\left(\xi_{i}\right)-\frac{1}{4}\right]^{2} } \\
\geq & {\left[\rho_{F}\left(\xi_{i}\right)-\frac{1}{4}\right]^{2}+\left[v_{F}\left(\xi_{i}\right)-\frac{1}{4}\right]^{2}+\left[\eta_{F}\left(\xi_{i}\right)-\frac{1}{4}\right]^{2}+\left[\phi_{F}\left(\xi_{i}\right)-\frac{1}{4}\right]^{2} }
\end{aligned}
$$

Proof: If $\rho_{E}\left(\xi_{i}\right) \leq \rho_{F}\left(\xi_{i}\right), v_{E}\left(\xi_{i}\right) \leq v_{F}\left(\xi_{i}\right)$ and $\eta_{E}\left(\xi_{i}\right) \leq \eta_{F}\left(\xi_{i}\right)$ with $\frac{1}{4} \geq \max \left\{\rho_{F}\left(\xi_{i}\right), \rho_{F}\left(\xi_{i}\right), \eta_{F}\left(\xi_{i}\right)\right\}$ then $\rho_{E}\left(\xi_{i}\right) \leq \rho_{F}\left(\xi_{i}\right) \leq \frac{1}{4}, v_{E}\left(\xi_{i}\right) \leq v_{F}\left(\xi_{i}\right) \leq \frac{1}{4}, \eta_{E}\left(\xi_{i}\right) \leq \eta_{F}\left(\xi_{i}\right) \leq \frac{1}{4}$ and $\phi_{E}\left(\xi_{i}\right) \leq \phi_{F}\left(\xi_{i}\right) \geq \frac{1}{4}$ which shows that (4.8) and (4.9) hold. Similarly, if $\rho_{E}\left(\xi_{i}\right) \geq \rho_{F}\left(\xi_{i}\right), v_{E}\left(\xi_{i}\right) \geq v_{F}\left(\xi_{i}\right), \eta_{E}\left(\xi_{i}\right) \geq \eta_{F}\left(\xi_{i}\right) \leq \frac{1}{4} \quad$ with $\max$ $\left\{\rho_{F}\left(\xi_{i}\right), v_{F}\left(\xi_{i}\right), v_{F}\left(\xi_{i}\right) \geq \frac{1}{4}\right\}$, then (4.8) and (4.9) hold. Szmidt and Kacpryzk [30] proposed the distance between two IFSs as the distance between their parametres, that is $(\rho, \mu, \eta)$. To determine the distance between two IFSs we uses Euclidean or Hamming distance measure.We may concluded from proposition (4.1) PFS $F$ is closer to maximum value $\left(\frac{1}{4}, \frac{1}{4}, \frac{1}{4}, \frac{1}{4}\right)$ than PFS $E$.

Theorem 4.2 Proposed entropy measure $V_{P F S}(E)$ is a valid PF measure.

Proof: To show the validity of $V_{P F S}(E)$, we have to prove the properties of definition (3.3).

W1: Let set $E$ is crisp. Then, it is possible if the values are either $\rho_{E}\left(\xi_{i}\right)=1$, and $v_{E}\left(\xi_{i}\right)=\eta_{E}\left(\xi_{i}\right)=$ $\phi_{E}\left(\xi_{i}\right)=0$ or $v_{E}\left(\xi_{i}\right)=1$, and $\rho_{E}\left(\xi_{i}\right)=\eta_{E}\left(\xi_{i}\right)=\phi_{E}\left(\xi_{i}\right)=0$ or $\eta_{E}\left(\xi_{i}\right)=1$ and $\rho_{E}\left(\xi_{i}\right)=v_{E}\left(\xi_{i}\right)=\phi_{E}\left(\xi_{i}\right)=0$ or $\phi_{E}\left(\xi_{i}\right)=1$ and $\rho_{E}\left(\xi_{i}\right)=v_{E}\left(\xi_{i}\right)=\eta_{E}\left(\xi_{i}\right)=0$.

$$
\Rightarrow\left(\rho_{E}\left(\xi_{i}\right)^{\frac{1}{2}}+v_{E}\left(\xi_{i}\right)^{\frac{1}{2}}+\eta_{E}\left(\xi_{i}\right)^{\frac{1}{2}}+\phi_{E}\left(\xi_{i}\right)^{\frac{1}{2}}\right)-\left(\rho_{E}\left(\xi_{i}\right)^{2}+v_{E}\left(\xi_{i}\right)^{2}+\eta_{E}\left(\xi_{i}\right)^{2}+\phi_{E}\left(\xi_{i}\right)^{2}\right)=0 .
$$

Therefore, $V_{P F S}(E)=0$.

Conversely, if $V_{P F S}(E)=0$, we have

$$
\left(\rho_{E}\left(\xi_{i}\right)^{\frac{1}{2}}+v_{E}\left(\xi_{i}\right)^{\frac{1}{2}}+\eta_{E}\left(\xi_{i}\right)^{\frac{1}{2}}+\phi_{E}\left(\xi_{i}\right)^{\frac{1}{2}}\right)-\left(\rho_{E}\left(\xi_{i}\right)^{2}+v_{E}\left(\xi_{i}\right)^{2}+\eta_{E}\left(\xi_{i}\right)^{2}+\phi_{E}\left(\xi_{i}\right)^{2}\right)=0,
$$

which is is possible under the following conditions:

1. either $\rho_{E}\left(\xi_{i}\right)=1$ and $v_{E}\left(\xi_{i}\right)=\eta_{E}\left(\xi_{i}\right)=\phi_{E}\left(\xi_{i}\right)=0$ or

2. $v_{E}\left(\xi_{i}\right)=1$, and $\rho_{E}\left(\xi_{i}\right)=\eta_{E}\left(\xi_{i}\right)=\phi_{E}\left(\xi_{i}\right)=0$ or

3. $\eta_{E}\left(\xi_{i}\right)=0$ and $\rho_{E}\left(\xi_{i}\right)=v_{E}\left(\xi_{i}\right)=\phi_{E}\left(\xi_{i}\right)=0$ or

4. $\phi_{E}\left(\xi_{i}\right)=1$ and $\rho_{E}\left(\xi_{i}\right)=v_{E}\left(\xi_{i}\right)=\eta_{E}\left(\xi_{i}\right)=0$.

Therefore we can say that $E$ is a crisp set iff $V_{P F S}(E)=0$.

W2: Since $\rho_{E}\left(\xi_{i}\right)+v_{E}\left(\xi_{i}\right)+\eta_{E}\left(\xi_{i}\right)+\phi_{E}\left(\xi_{i}\right)=1$, to get the maximum value of PF entropy $V_{P F S}(E)$, we uses the method of Lagrange's method of undetermined multiplier.We write $g\left(\rho_{E}, v_{E}, \phi_{E}\right)=\rho_{E}\left(\xi_{i}\right)+v_{E}\left(\xi_{i}\right)+$ $\eta_{E}+\phi_{E}\left(\xi_{i}\right)-1$. Let

$$
G\left(\rho_{E}, v_{E}, \phi_{E}\right)=V_{P F S}\left(\rho_{E}, v_{E}, \eta_{E}, \phi_{E}\right)+\mu g\left(\rho_{E}, v_{E}, \eta_{E}, \phi_{E}\right)
$$

where $\mu$ is Lagrange's multiplier. Type equation here. Differentiating (4.10) partially w.r.t. $\rho_{M}, v_{M}, \eta_{M}, \phi_{M}$ and $\mu$ and putting equal to zero, we get $\rho_{E}\left(\xi_{i}\right)=v_{E}\left(\xi_{i}\right)=\eta_{E}\left(\xi_{i}\right)=\phi_{E}\left(\xi_{i}\right)=\frac{1}{4}$. The stationary point of $V_{P F S}(E)$ is $\rho_{E}\left(\xi_{i}\right)=v_{E}\left(\xi_{i}\right)=\eta_{E}\left(\xi_{i}\right)=\phi_{E}\left(\xi_{i}\right)=\frac{1}{4}$. By using Hessian matrix we can prove that $V_{P F S}(E)$ is a concave function at the stationary points .

Definition 4.1 (Hessian) The Hessian matrix of a function $\varphi\left(\mathrm{x}_{1}, \mathrm{x}_{2}, \mathrm{x}_{3}, \mathrm{x}_{4}\right)$ of four variables is given by 


$$
[\operatorname{HEN}\rceil(\varphi)=\left[\begin{array}{cccc}
\frac{\partial^{2} \varphi}{\partial x_{1}^{2}} & \frac{\partial^{2} \varphi}{\partial x_{2} \partial x_{1}} & \frac{\partial^{2} \varphi}{\partial x_{3} \partial x_{1}} & \frac{\partial^{2} \varphi}{\partial x_{4} \partial x_{1}} \\
\frac{\partial^{2} \varphi}{\partial x_{1} \partial x_{2}} & \frac{\partial^{2} \varphi}{\partial x_{2}^{2}} & \frac{\partial^{2} \varphi}{\partial x_{3} \partial x_{2}} & \frac{\partial^{2} \varphi}{\partial x_{4} \partial x_{2}} \\
\frac{\partial^{2} \varphi}{\partial x_{1} \partial x_{3}} & \frac{\partial^{2} \varphi}{\partial x_{2} \partial x_{3}} & \frac{\partial^{2} \varphi}{\partial x_{3}^{2}} & \frac{\partial^{2} \varphi}{\partial x_{4} \partial x_{3}} \\
\frac{\partial^{2} \varphi}{\partial x_{1} \partial x_{4}} & \frac{\partial^{2} \varphi}{\partial x_{2} \partial x_{4}} & \frac{\partial^{2} \varphi}{\partial x_{3} \partial x_{4}} & \frac{\partial^{2} \varphi}{\partial x_{4}^{2}}
\end{array}\right]
$$

$\varphi$ is said to be strictly concave if If $[\operatorname{HEN}\rceil(\varphi)$ is negative definite at a point in its domain and The Hessian of $V_{P F S}(E)$ is given by

$$
\lceil\operatorname{HEN}\rceil\left(\mathrm{V}_{\mathrm{PFS}}(\mathrm{E})\right)=\mathrm{b}\left[\begin{array}{rrrr}
-1 & 0 & 0 & 0 \\
0 & -1 & 0 & 0 \\
0 & 0 & -1 & 0 \\
0 & 0 & 0 & -1
\end{array}\right]
$$

where

$b=\left(\frac{1}{4 \rho_{E}\left(\xi_{i}\right)^{\frac{3}{2}}}+2\right)\left(\frac{1}{4 v_{E}\left(\xi_{i}\right)^{\frac{3}{2}}}+2\right)\left(\frac{1}{\eta_{E}\left(\xi_{i}\right)^{\frac{3}{2}}}+2\right)\left(\frac{1}{4 \phi_{E}\left(\xi_{i}\right)^{\frac{3}{2}}}+2\right), \mathrm{b}$ is positive at maximum value of stationary point are $\rho_{E}\left(\xi_{i}\right)=v_{E}\left(\xi_{i}\right)=\eta_{E}\left(\xi_{i}\right)=\phi_{E}\left(\xi_{i}\right)=\frac{1}{4}$. Thus $\lceil\operatorname{HEN}\rceil\left(\mathrm{V}_{\mathrm{PFS}}(\mathrm{E})\right)$ is negative definite so $\mathrm{V}_{\mathrm{PFS}}(\mathrm{E})$ is strictly concave.

W3: For any PFS, $V_{P F S}(E)=V_{P F S}\left(E^{c}\right)$, which is clear from the definition.

W4: Since, $V_{P F S}(E)$ has maximum value at stationary point is also a concave function, if $\max \left\{\rho_{E}\left(\xi_{i}\right), v_{E}\left(\xi_{i}\right), \eta_{E}\left(\xi_{i}\right), \phi_{E}\left(x_{I}\right)\right\} \leq \frac{1}{4}$, then $\rho_{E}\left(\xi_{i}\right) \leq \rho_{F}\left(\xi_{i}\right), v_{E}\left(\xi_{i}\right) \leq v_{F}\left(\xi_{i}\right)$ and $\eta_{E}\left(\xi_{i}\right) \leq \eta_{F}\left(\xi_{i}\right)$ implies $\phi_{E}\left(\xi_{i}\right) \geq \phi_{F}\left(\xi_{i}\right) \geq \frac{1}{4}$. Therefore, by using proposition (4.1), we see that $V_{2}(E)$ satisfies the condition $W 4$.

If $\min \left\{\rho_{E}\left(\xi_{i}\right), v_{E}\left(\xi_{i}\right), \eta_{E}\left(\xi_{i}\right)\right\} \geq \frac{1}{4}$,then $\rho_{E}\left(\xi_{i}\right) \leq \rho_{F}\left(\xi_{i}\right), v_{E}\left(\xi_{i}\right) \geq v_{F}\left(\xi_{i}\right)$ and $\eta_{E}\left(\xi_{i}\right) \geq \eta_{F}\left(\xi_{i}\right)$. Again, by using proposition (4.1), we observe that $V_{P F S}(E)$ gratifies condition $W 4$.

Theorem 4.3 For any two $E_{1}, E_{2} \in P F S\left(Y^{*}\right)$, such that for all $\xi_{i} \in Y^{*}$ either $E_{1} \subseteq E_{2}$ or $E_{2} \subseteq E_{1}$; then,

$$
V_{P F S}\left(E_{1} \cup E_{2}\right)+V_{P F S}\left(E_{1} \cap E_{2}\right)=V_{P F S}\left(E_{1}\right)+V_{P F S}\left(E_{2}\right)
$$

Proof. To prove this theorm, let $Y^{*}$ be divided into $Y_{1}^{*}$ and $Y_{2}^{*}$, such that

$$
\begin{gathered}
Y_{1}^{*}=\left\{\xi_{i} \in Y^{*}: E_{1} \subseteq E_{2}\right\}, \text { and } Y_{2}^{*}=\left\{\xi_{i} \in Y^{*}: E_{2} \subseteq E_{1}\right\} \\
\rho_{E_{1}}\left(\xi_{i}\right) \leq \rho_{E_{2}}\left(\xi_{i}\right), v_{E_{1}}\left(\xi_{i}\right) \leq v_{E_{2}}\left(\xi_{i}\right), \eta_{E_{1}}\left(\xi_{i}\right) \geq \eta_{E_{2}}\left(\xi_{i}\right) \quad \forall \quad \xi_{i} \in Y^{*} \\
\rho_{E_{1}}\left(\xi_{i}\right) \geq \rho_{E_{2}}\left(\xi_{i}\right), v_{E_{1}}\left(\xi_{i}\right) \geq v_{E_{2}}\left(\xi_{i}\right), \eta_{E_{1}}\left(\xi_{i}\right) \geq \eta_{E_{2}}\left(\xi_{i}\right) \quad \forall \quad \xi_{i} \in Y^{*} \\
\text { Now, } \quad V_{P F S}\left(E_{1} \cup E_{2}\right)=\frac{2}{3 n} \sum_{i=1}^{n}\left[\left(\rho_{\left(E_{1} \cup E_{2}\right)}\left(\xi_{i}\right)^{\frac{1}{2}}+v_{\left(E_{1} \cup E_{2}\right)}\left(\xi_{i}\right)^{\frac{1}{2}}+\eta_{\left(E_{1} \cup E_{2}\right)}\left(\xi_{i}\right)^{\frac{1}{2}}\right.\right. \\
\left.\left.+\phi_{\left(E_{1} \cup E_{2}\right)}\left(\xi_{i}\right)^{\frac{1}{2}}\right)-\left(\rho_{\left(E_{1} \cup E_{2}\right)}\left(\xi_{i}\right)^{2}+v_{\left(E_{1} \cup E_{2}\right)}\left(\xi_{i}\right)^{2}+\eta_{\left(E_{1} \cup E_{2}\right)}\left(\xi_{i}\right)^{2}+\phi_{\left(E_{1} \cup E_{2}\right)}\left(\xi_{i}\right)^{2}\right)\right] \\
=\frac{2}{3 n} \sum_{Y_{1}^{*}}\left[\left(\rho_{E_{2}}\left(\xi_{i}\right)^{\frac{1}{2}}+v_{E_{2}}\left(\xi_{i}\right)^{\frac{1}{2}}+\phi_{E_{2}}\left(\xi_{i}\right)^{\frac{1}{2}}-\left(\rho_{E_{2}}\left(\xi_{i}\right)^{2}+v_{E_{2}}\left(\xi_{i}\right)^{2}+\phi_{E_{2}}\left(\xi_{i}\right)^{2}\right)\right)\right] \\
+\frac{2}{3 n} \sum_{Y_{2}^{*}}\left[\left(\rho_{E_{1}}\left(\xi_{i}\right)^{\frac{1}{2}}+v_{E_{1}}\left(\xi_{i}\right)^{\frac{1}{2}}+\phi_{E_{1}}\left(\xi_{i}\right)^{\frac{1}{2}}\right)-\left(\rho_{E_{1}}\left(\xi_{i}\right)^{2}+v_{E_{1}}\left(\xi_{i}\right)^{2}+\phi_{E_{1}}\left(\xi_{i}\right)^{2}\right)\right]
\end{gathered}
$$

Similarly, we get

$$
\begin{gathered}
V_{P F S}\left(E_{1} \cap E_{2}\right) \\
=\frac{2}{3 n} \sum_{Y_{1}^{*}}\left[\left(\rho_{E_{1}}\left(\xi_{i}\right)^{\frac{1}{2}}+v_{E_{1}}\left(\xi_{i}\right)^{\frac{1}{2}}+\phi_{E_{1}}\left(\xi_{i}\right)^{\frac{1}{2}}-\left(\rho_{E_{1}}\left(\xi_{i}\right)^{2}+v_{E_{1}}\left(\xi_{i}\right)^{2}+\phi_{E_{1}}\left(\xi_{i}\right)^{2}\right)\right)\right] \\
+\frac{2}{3 n} \sum_{Y_{2}^{*}}\left[\left(\rho_{E_{2}}\left(\xi_{i}\right)^{\frac{1}{2}}+v_{E_{2}}\left(\xi_{i}\right)^{\frac{1}{2}}+\phi_{E_{2}}\left(\xi_{i}\right)^{\frac{1}{2}}\right)-\left(\rho_{E_{2}}\left(\xi_{i}\right)^{2}+v_{E_{2}}\left(\xi_{i}\right)^{2}+\phi_{E_{2}}\left(\xi_{i}\right)^{2}\right)\right]
\end{gathered}
$$

Now, adding (4.17)and (4.18), we have 


$$
V_{P F S}\left(E_{1} \cup E_{2}\right)+V_{P F S}\left(E_{1} \cap E_{2}\right)=V_{P F S}\left(E_{1}\right)+V_{P F S}\left(E_{2}\right)
$$

This proves the theorem.

Corollary 4.1. For any Picture fuzzy set $E$ and $E^{c}$ (complement of $E$ ), we have

$$
V_{P F S}(E)=V_{P F S}\left(E^{c}\right)=V_{P F S}\left(E \cup E^{c}\right)+V_{P F S}\left(E \cap E^{c}\right) \text {. }
$$

\section{An extension of TODIM based on VIKOR for Picture Fuzzy MCDM Problem}

In this section we will use hybrid TODIM-VIKOR method to MCDM problems for opinion poll based on the proposed entropy measure for PFSs. To show validity and practical reasonability, we apply proposed measure in a MCDM problem, involving partially known and unknown information about criteria weights for alternatives in PF information.

Let us consider a case of nation where elections will be held in near future. Let $\mathrm{P}_{1}, \mathrm{P}_{2}, \mathrm{P}_{3}, \mathrm{P}_{4}, \mathrm{P}_{5}$ are are different political parties are contesting and they are contesting on different issues say: (1) National security (2) economy (3) employment (4) stability (5) corruption. A survey on 1000 people has been conducted by news channel for opinion poll to determine the possible outcomes of elections. To get the best possible outcome we applied the PF TODIM-VIKOR approaches to this kind of problems with PFNs, and the procedure for hybrid TODIM-VIKOR method is as follows :

\subsection{VIKOR Method}

The idea of VIKOR was given Opricovic et al. [26] to determine a compromise solution which is very near to the consistent solution. This solution is helpful to find the best solution by taking the majority and minimize the (“opponent") with conflicting criteria.

By considering alternative $\mathrm{P}_{i}$ corresponding to each critera $\Gamma_{j}$ is given as

$\gamma_{i j}(1 \leq i \leq m$ and $1 \leq j \leq n)$. Improved VIKOR method by Yu PL[13] is given by

$$
l_{p, i}=\left\{\sum_{j=1}^{n}\left(w_{j} \frac{\left(D_{j}^{+}-\widehat{D}_{i j}\right)}{\left(D_{j}^{+}-\widehat{D}_{j}^{-}\right)}\right)^{p}\right\}^{\frac{1}{p}}, 1 \leq p \leq \infty, 1 \leq i \leq m ;
$$

where $D_{j}^{+}=\max _{i} \widehat{D}_{i j}$ and $D_{j}^{-}=\min _{i} \widehat{D}_{i j}$ givs the best and worst solutions. $w_{j}$ shows the weight criteria and $l_{p, i}$ gives the distance of alternative $P_{i}$ to the best solution.In the VIKOR method $l_{1, j}\left(\operatorname{as} S_{i}\right)$ and $l_{\infty, i}\left(\operatorname{as} G_{i}\right), i=$ $1,2, \ldots m$ are used to formulate as "boundary measures". The main steps for the new PF TODIM-VIKOR method based on the proposed entropy measure as follows .

Step 1: Consider the alternative $\mathrm{P}_{i}$ acting on the criterion $\Gamma_{j}$ is denoted in terms of picture fuzzy value (PFV) $\gamma_{i j}=\left(\rho_{i j}, \mu_{i j}, \eta_{i j}\right) ; 1 \leq i \leq m, 1 \leq j \leq n$. We design a Picture fuzzy decision matrix $\widehat{D}=\left[\gamma_{i j}\right]_{m \times n}$ as follows:

$$
D=\left[d_{i j}\right]_{m \times n}=\left[\begin{array}{ccccc} 
& \Gamma_{1} & \Gamma_{2} & \ldots & \Gamma_{\mathrm{m}} \\
\mathrm{p}_{1} & \left(\rho_{\beta_{11}}, \mu_{\beta_{11}}, \eta_{\beta_{11}}\right) & \left(\rho_{\beta_{12}}, \mu_{\beta_{12}}, \eta_{\beta_{12}}\right) & \ldots & \left(\rho_{\beta_{1 n}}, \mu_{\beta_{1 n}}, \eta_{\beta_{1 n}}\right) \\
P_{2} & \left(\rho_{\beta_{21}}, \mu_{\beta_{21}}, \eta_{\beta_{21}}\right) & \left(\rho_{\beta_{22}}, \mu_{\beta_{22}}, \eta_{\beta_{22}}\right) & \ldots & \left(\rho_{\beta_{2 n}}, \mu_{\beta_{2 n}}, \eta_{\beta_{2 n}}\right) \\
\vdots & \vdots & \vdots & \ddots & \vdots \\
P_{\mathrm{m}} & \left(\rho_{\beta_{m 1}}, \mu_{\beta_{m 1}}, \eta_{\beta_{m 1}}\right) & \left(\rho_{\beta_{m 2}}, \mu_{\beta_{m 2}}, \eta_{\beta_{m 2}}\right) & \ldots & \left(\rho_{\beta_{m n}}, \mu_{\beta_{m n}}, \eta_{\beta_{m n}}\right)
\end{array}\right]
$$

Step 2:Convert the decision matrix $\widehat{D}=\left(\gamma_{i j}\right)_{m \times n}$ into a normalized PF decision matrix which is denoted as :

$$
q_{i j}=\left(\begin{array}{ll}
\left(\gamma_{i j}\right)^{c}, & \text { for cost criteria } \\
\gamma_{i j}, & \text { for benefit criteria }
\end{array}\right.
$$

where $\gamma_{i j}{ }^{c}=\left(\eta_{i j}, \mu_{i j}, \rho_{i j}\right)$ is complement of $\gamma_{i j}$. After that we will obtain a new PF decision matrix $D=$ $\left(q_{i j}\right)_{m \times n}$

Step 3:

Methodology 1: If the criteria weights are partially known 
We will solve the MCDM problem if the attribute weight are partially known and also completely known by collecting all PF information under distinct conditions and then we will compare the final PF values. Here we used the proposed measure to find the attribute weights by using the below said formula:

$$
\begin{gathered}
\operatorname{Min} \hat{T}=\sum_{j=1}^{n} w_{j} \sum_{i=1}^{m} V_{P F S}\left(\rho_{i j}\right) \\
\text { such that } w_{j} \geq 0,1 \leq j \leq n, \sum_{j=1}^{n} w_{j}=1
\end{gathered}
$$

\section{Methodology 2: When the Criteria Weights are completely unknown}

The attribute weight can be calculated by using the below said equation if attribute weights are completely unknown.

$$
w_{j}=\frac{\sum_{i=1}^{m} V_{P F S}\left(\rho_{i j}\right)}{\sum_{j=1}^{n} \sum_{i=1}^{m} V_{P F S}\left(\rho_{i j}\right)}
$$

Determine the vlaue of $\mathrm{P}_{i}$ corresponding to each criterion $\Gamma_{j}$ based on $w=\left(w_{1}, w_{2}, \ldots, w_{n}\right)^{T}$ as:

$$
w_{j r}=\frac{w_{j}}{w_{r}}, j, r=1,2, \ldots, n
$$

where $w_{j}$ is the weight of the criterion $\Gamma_{j}, w_{r}=\max \left\{w_{1}, w_{2}, \ldots, w_{n}\right\}$ and $0 \leq w_{j r} \leq 1$. In this step, each criteria carries equal importance, any criterion may be choosen.

\section{Step 4:}

\section{TODIM Method}

The TODIM method is a discrete multicriteria method used for qualitative as well as quantitative criteria based on probability theory. The dominance degree of $\mathrm{P}_{i}$ over each alternative $\mathrm{P}_{j}$ w.r.t. each criterion $\Gamma_{j}$ is given by:

$$
Z_{j}\left(\mathrm{P}_{i}, \mathrm{P}_{\left.t_{1}\right)}=\left(\begin{array}{ll}
\sqrt{\frac{w_{j r} d_{H}\left(q_{i j}, q_{t_{1 j}}\right)}{\sum_{j=1}^{n} w_{j r}}}, & \text { if } q_{i j}>q_{t_{1} j} \\
0, & \text { if } q_{i j}=q_{t_{1} j} \\
-\frac{1}{r} \sqrt{\frac{\left(\sum_{j=1}^{n} w_{j r}\right) d_{H}\left(q_{i j}, q_{t_{1} j}\right)}{w_{j r}}}, & \text { if } q_{i j}<q_{t_{1} j}
\end{array}\right.\right.
$$

where $d_{H}\left(q_{i j}, q_{t_{1} j}\right)$ find the distance between the two PFNs $q_{i j}$ and $q_{t_{1} j}$ and the parameter $\gamma$ represents the attenuation factor of losses. By definition if $q_{i j}>q_{t_{1 j} j}$, then $Z_{j}\left(\mathrm{P}_{i}, \mathrm{P}_{t_{1}}\right)$ signifies a gain ; if $q_{i j}<q_{t_{1 j} j}$, then $Z_{j}\left(\mathrm{P}_{i}, \mathrm{P}_{t_{1}}\right)$ signifies loss.

Step 5: For each alternative $\mathrm{P}_{i}$, the dominance matrix is shown below :

$$
Z_{j}=\left[Z_{j}\left(r_{i}, r_{t_{1}}\right)\right]_{m \times m}=\left[\begin{array}{ccccc} 
& \Gamma_{1} & \Gamma_{2} & \ldots & \Gamma_{\mathrm{m}} \\
\mathrm{P}_{1} & 0 & Z_{j}\left(R_{1}, R_{2}\right) & \ldots & Z_{j}\left(R_{1}, R_{\mathrm{m}}\right) \\
\mathrm{P}_{2} & Z_{j}\left(R_{2}, R_{1}\right) & 0 & & Z_{j}\left(R_{2}, R_{\mathrm{m}}\right) \\
\vdots & \vdots & \vdots & \ddots & \vdots \\
\mathrm{P}_{\mathrm{m}} & Z_{j}\left(R_{m}, R_{1}\right) & Z_{j}\left(R_{m}, R_{2}\right) & \ldots & 0
\end{array}\right]
$$

Step 6: The total dominance degree of each alternative $\mathrm{P}_{i}$ w.r.t. another alternatives $\mathrm{P}_{t_{1}}\left(1 \leq t_{1} \leq \mathrm{m}\right)$ is given by :

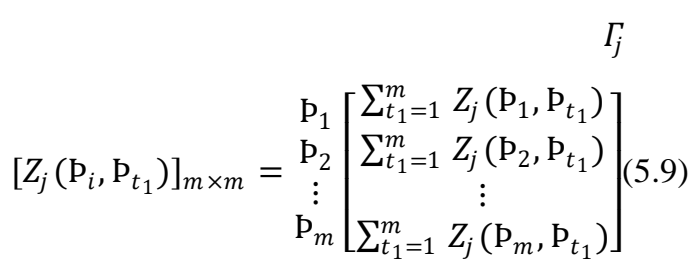

From the set of n-criteria got as $\widehat{D}$ shows the dominance matrix: 


$$
\left[t_{i j}\right]_{m \times r}=\begin{array}{r}
\mathrm{P}_{1} \\
\mathrm{P}_{2} \\
\vdots \\
\mathrm{P}_{m}
\end{array}\left[\begin{array}{ccccc}
\sum_{t_{1}=1}^{m} Z_{1}\left(\mathrm{P}_{1}, \mathrm{P}_{t_{1}}\right) & \sum_{t_{1}=1}^{m} Z_{2}\left(\mathrm{P}_{1}, \mathrm{P}_{t_{1}}\right) & \ldots & \sum_{t_{1}=1}^{m} Z_{r}\left(\mathrm{P}_{1}, \mathrm{P}_{t_{1}}\right) \\
\sum_{t_{1}=1}^{m} Z_{1}\left(\mathrm{P}_{2}, \mathrm{P}_{t_{1}}\right) & \sum_{t_{1}=1}^{m} Z_{2}\left(\mathrm{P}_{2}, \mathrm{p}_{t_{1}}\right) & \ldots & \sum_{t_{1}=1}^{m} Z_{r}\left(\mathrm{P}_{2}, \mathrm{p}_{t_{1}}\right) \\
\vdots & \vdots & \ddots & & \vdots \\
\left.\sum_{t_{1}=1}^{m} Z_{1}\left(\mathrm{P}_{m}, \mathrm{P}_{t_{1}}\right)\right) & \sum_{t_{1}=1}^{m} Z_{2}\left(\mathrm{P}_{m}, \mathrm{P}_{t_{1}}\right) & \ldots & \sum_{t_{1}=1}^{m} Z_{r}\left(\mathrm{P}_{m}, \mathrm{p}_{t_{1}}\right)
\end{array}\right]
$$

Step 7: $\widehat{D}^{+}$shows the (best value )or positive solution and $D^{-}$(worst value) or negative solution:

$$
\begin{gathered}
\widehat{D}^{+}=\left(\widehat{D}_{1}^{+}, \widehat{D}_{2}^{+}, \ldots, \widehat{D}_{n}^{+}\right) \\
=\left(\max \sum_{i, t_{1}=1}^{m} Z_{1}\left(\mathrm{P}_{i}, \mathrm{P}_{t_{1}}\right), \max \sum_{i, t_{1}=1}^{m} Z_{2}\left(\mathrm{P}_{i}, \mathrm{P}_{t_{1}}\right), \ldots, \max \sum_{i, t_{1}=1}^{m} Z_{n}\left(\mathrm{P}_{i}, \mathrm{P}_{t_{1}}\right)\right) \\
\operatorname{and} \widehat{D}^{-}=\left(\widehat{D}_{1}^{-}, \widehat{D}_{2}^{-}, \ldots, \widehat{D}_{n}^{-}\right) \\
=\left(\min \sum_{i, t_{1}=1}^{m} Z_{1}\left(\mathrm{P}_{i}, \mathrm{P}_{t_{1}}\right), \min \sum_{i, t_{1}=1}^{m} Z_{2}\left(\mathrm{P}_{i}, \mathrm{P}_{t_{1}}\right), \ldots, \min \sum_{i, t_{1}=1}^{m} Z_{n}\left(\mathrm{P}_{i}, \mathrm{P}_{t_{1}}\right)\right)
\end{gathered}
$$

Step 8: Consider $L_{i}$ as "maximum cluster usefulness " and $G_{i}$ as "minimum of the individual remorse value", determine the compromise solution as :

$$
\begin{aligned}
L_{i} & =\sum_{1 \leq j \leq n} w_{j} \frac{d_{H}\left(D_{j}^{+}, \widehat{D}_{i j}\right)}{d_{H}\left(D_{j}^{+}, \widehat{D}_{j}^{-}\right)} \\
G_{i} & =\max _{1 \leq j \leq n}\left(w_{j} \frac{d_{H}\left(D_{j}^{+}, \widehat{D}_{i j}\right)}{d_{H}\left(D_{j}^{+}, \widehat{D}_{j}^{-}\right)}\right)
\end{aligned}
$$

where

$$
\begin{aligned}
& d_{H}\left(D_{j}^{+}, \widehat{D}_{i j}\right)=\max _{1 \leq i \leq n} \sum_{t_{1}=1}^{m} Z_{j}\left(\mathrm{P}_{i}, \mathrm{P}_{t_{1}}\right)-\sum_{t_{1}=1}^{m} Z_{j}\left(\mathrm{P}_{i}, \mathrm{P}_{t_{1}}\right), \\
& d_{H}\left(D_{j}^{+}, \widehat{D}_{j}\right)=\max _{1 \leq i \leq n} \sum_{t_{1}=1}^{m} Z_{j}\left(\mathrm{P}_{i}, \mathrm{P}_{t_{1}}\right)-\min _{1 \leq i \leq n} \sum_{t_{1}=1}^{m} Z_{j}\left(\mathrm{P}_{i}, \mathrm{P}_{t_{1}}\right)
\end{aligned}
$$

and $w_{j} ;(j=1,2, \ldots, m)$ shows the weight of $j^{\text {th }}$ criteria with $\sum_{j=1}^{n} w_{j}=1$.

Step 9:Compute the influence index $M_{i}, i=1,2, \ldots, m$ with Equation (5.13).

$$
M_{i}=\xi \frac{L_{i}-L^{-}}{\bar{L}-L^{-}}+(1-\xi) \frac{G_{i}-G^{-}}{\bar{G}-G^{-}} .
$$

where $L^{-}=\min _{i}\left(L_{i}\right), \bar{L}=\max _{i}\left(L_{i}\right), \bar{G}=\max _{i}\left(G_{i}\right)$ and $G^{-}=\min _{i}\left(G_{i}\right)$. The coefficient $\xi$ and $1-\xi$ denote the weight-age assigned to $\left(L_{i}\right)$ and $\left(G_{i}\right)$. In general, we set the value to $\xi=\frac{1}{2}$ and $\xi=\frac{1}{2}$ denotes a consensus.

Step 10:Assigned rank to the alternatives by arranging the values of $\left(L_{i}\right),\left(G_{i}\right)$ and $\left(M_{i}\right)(i=1,2, \ldots, m)$ in ascending order.

Step 11:Then find the compromise solution, if the solution satisfies the following two conditions will be the most desirable solution.

$\mathbf{X 1}$ :If $Q\left(\mathrm{P}^{(2)}\right)-Q\left(\mathrm{P}^{(1)}\right) \geq \frac{1}{n-1}$, where $\mathrm{P}^{(1)}$ and $\mathrm{P}^{(2)}$, respectively, placed at first and second positions in the table of $Q_{i}$ and $n$ shows the number of criteria.

X2: The alternative $\mathrm{P}^{(1)}$ is placed at first positin in the ranking of the values of $L_{i}$ or /and $G_{i}$. This compromise solution is consistent within a decision making process, which is : voting by majority rule (if $\xi>0.5$ ), or with veto (if $\xi<0.5$ ),or by conseness (if $\xi=0.5$ ).

If the conditions $\mathrm{X} 1$ and $\mathrm{X} 2$ are not simultaneously satisfied, at that point we look for the compromise solution as follows:

(a) (Acceptable advantage):, The alternatives $\mathrm{P}^{(1)}$ and $\mathrm{P}^{(2)}$ gives the compromised solutions If only condition $\mathrm{X} 2$ is not satisfied.

(b) (Acceptable stability): The alternatives $\mathrm{P}^{(1)}, \mathrm{P}^{(2)}, \ldots, \mathrm{P}^{(A)}$ will be the compromise solution ; $\mathrm{p}^{(A)}$ is calculated by the equation

$$
M\left(\mathrm{P}^{(A)}\right)-Q\left(\mathrm{P}^{(1)}\right)<\frac{1}{A-1}
$$

for maximum $E$ If the condition X1 is not satisfied. 


\section{Method 1:For Partially Known Criteria Weights}

The PF decision matrix depicted in table 2 shows the overall views of the public. To find the PFN's $\left(\rho_{i j}, v_{i j}, \eta_{i j}\right)$ we proceed as follows:

Since 1000 people have been considered for survey , consider 300 people support the party $\mathrm{P}_{1}$ corresponding to criteria $\Gamma_{1}$ that is "yes", 200 people are neutral and 100 people do not support the party $\mathrm{P}_{1}$ or say no. Then, the $\operatorname{PFN}\left(\rho_{11}, v_{11}, \eta_{11}\right)$ is given by

$$
\rho_{11}=\frac{30}{1000}=.30, v_{11}=\frac{20}{1000}=.20, \eta_{11}=\frac{10}{1000}=.10
$$

In a similar way, we can obtain other entries of PF decision matrix.

\section{Results study with proposed method in PF framework:}

Step 1.

\begin{tabular}{|c|c|c|c|c|c|}
\hline $\begin{array}{c}\text { Decision } \\
\text { value }\end{array}$ & $\Gamma_{1}$ & $\Gamma_{2}$ & $\Gamma_{3}$ & $\Gamma_{4}$ & $\Gamma_{5}$ \\
\hline $\mathrm{P}_{1}$ & $(0.3,0.2,0.1)$ & $(0.7,0.1,0.1)$ & $(0.1,0.2,0.6)$ & $(0.4,0.1,0.4)$ & $(0.1,0.4,0.2)$ \\
\hline $\mathrm{P}_{2}$ & $(0.2,0.1,0.6)$ & $(0.5,0.3,0.1)$ & $(0.5,0.1,0.3)$ & $(0.4,0.3,0.2)$ & $(0.2,0.3,0.4)$ \\
\hline $\mathrm{P}_{3}$ & $(0.3,0.1,0.6)$ & $(0.2,0.4,0.2)$ & $(0.8,0.0,0.1)$ & $(0.1 .0 .4,0.2)$ & $(0.4,0.4,0.1)$ \\
\hline $\mathrm{P}_{4}$ & $(0.5,0.3,0.1)$ & $(0.5,0.2,0.2)$ & $(0.2,0.3,0.2)$ & $(0.2,0.1,0.6)$ & $(0.5,0.2,0.1)$ \\
\hline $\mathrm{P}_{5}$ & $(0.1,0.4,0.3)$ & $(0.2,0.6,0.1)$ & $(0.5,0.1,0.3)$ & $(0.6,0.1,0.1)$ & $(0.6,0.1,0.3)$ \\
\hline
\end{tabular}

Table 1: PF-decision matrix $\widehat{D}$

Step2. Since $\Gamma_{1}$ and $\Gamma_{4}$ are cost criterion and $\Gamma_{2}, \Gamma_{3}$ and $\Gamma_{5}$ are benefit criterion, then the table 2 shows the normalized decision matrix $\widehat{D}$.

\begin{tabular}{|c|c|c|c|c|c|}
\hline $\begin{array}{l}\text { Decision } \\
\text { value }\end{array}$ & $\Gamma_{1}$ & $\Gamma_{2}$ & $\Gamma_{3}$ & $\Gamma_{4}$ & $\Gamma_{5}$ \\
\hline $\mathrm{P}_{1}$ & $(0.1,0.2,0.3)$ & $(0.7,0.1,0.1)$ & $(0.1,0.2,0.6)$ & $(0.4,0.1,0.4)$ & $(0.1,0.4,0.2)$ \\
\hline $\mathrm{P}_{2}$ & $(0.6,0.1,0.2)$ & $(0.5,0.3,0.1)$ & $(0.5,0.1,0.3)$ & $(0.2,0.3,0.4)$ & $(0.2,0.3,0.4)$ \\
\hline $\mathrm{P}_{3}$ & $(0.6,0.1,0.3)$ & $(0.2,0.4,0.2)$ & $(0.8,0.0,0.1)$ & $(0.2,0.4,0.1)$ & $(0.4,0.4,0.1)$ \\
\hline $\mathrm{P}_{4}$ & $(0.1,0.3,0.5)$ & $(0.5,0.2,0.2)$ & $(0.2,0.3,0.2)$ & $(0.6,0.1,0.2)$ & $(0.5,0.2,0.1)$ \\
\hline$P_{5}$ & $(0.3,0.4,0.1)$ & $(0.2,0.6,0.1)$ & $(0.5,0.1,0.3)$ & $(0.1,0.1,0.6)$ & $(0.6,0.1,0.3)$ \\
\hline
\end{tabular}

Table 2: Picture Normalized fuzzy decision matrix $\widehat{D}$

Step 3. Let

$$
\begin{array}{ll}
\widehat{T}=\left\{0.12 \leq w_{1} \leq 0.26,0.17 \leq w_{2} \leq 0.19,\right. & 0.28 \leq w_{3} \leq 0.39,0.19 \leq w_{4} \leq 0.46, \\
& \left.0.10 \leq w_{5} \leq 0.16\right\} .
\end{array}
$$

The total entropy of each attribute are as follows:

$$
\begin{gathered}
K_{1}=\sum_{i=1}^{5} \rho_{1 j}=\sum_{i=1}^{5} V_{2}\left(q_{1 j}\right)=0.9903 ; K_{2}=\sum_{i=1}^{5} \rho_{2 j}=\sum_{i=1}^{5} V_{2}\left(q_{2 j}\right)=0.9998 ; \\
K_{3}=\sum_{i=1}^{5} \rho_{3 j}=\sum_{i=1}^{5} V_{2}\left(q_{3 j}\right)=0.9448 ; K_{4}=\sum_{i=1}^{5} \rho_{4 j}=\sum_{i=1}^{5} V_{2}\left(q_{4 j}\right)=1.0284 ; \\
K_{5}=\sum_{i=1}^{5} \rho_{5 j}=\sum_{i=1}^{5} V_{2}\left(q_{5 j}\right)=1.0134
\end{gathered}
$$

The following optimal model is used to find the attribute weights :

$\operatorname{Min} T=0.99034 w_{1}+0.9998 w_{2}+0.9448 w_{3}+1.0284 w_{4}+1.0134 w_{5}$

$$
\text { such that } w \in H \sum_{j=1}^{5} w_{j}=1 w_{j} \geq 0, j=1,2,3,4,5 \text {. }
$$

After solning the above equation we obtained the following weighting vector of the attribute: 


$$
w=(0.15,0.17,0.39,0.19,0.10)^{T} .
$$

. Step 4. We can form the dominance matrices $Z_{1}-Z_{5}$ by assuming the value of $\gamma=2.5$ from the table 3 :

$$
\begin{aligned}
& Z_{1}=\left[\begin{array}{rrlrrr} 
& \multicolumn{1}{c}{\Gamma_{1}} & \Gamma_{2} & \multicolumn{1}{c}{\Gamma_{3}} & \multicolumn{1}{l}{\Gamma_{4}} & \multicolumn{1}{c}{\Gamma_{5}} \\
\mathrm{P}_{1} & 0.0000 & 0.1871 & 0.1732 & -0.3266 & 0.1732 \\
\mathrm{P}_{2} & -0.4989 & 0.0000 & -0.1886 & -0.5963 & -0.4989 \\
\mathrm{P}_{3} & -0.4619 & 0.0707 & 0.0000 & -0.5657 & -0.5333 \\
\mathrm{P}_{4} & 0.1225 & 0.2236 & 0.2121 & 0.0000 & 0.1871 \\
\mathrm{P}_{5} & -0.4619 & 0.1871 & 0.2000 & -0.4989 & 0.0000
\end{array}\right] \\
& Z_{2}=\left[\begin{array}{rrrrrr} 
& \multicolumn{1}{l}{\Gamma_{1}} & \multicolumn{1}{l}{\Gamma_{2}} & \Gamma_{3} & \multicolumn{1}{l}{\Gamma_{4}} & \multicolumn{1}{l}{\Gamma_{5}} \\
\mathrm{P}_{1} & 0.0000 & 0.1505 & 0.2258 & 0.1505 & 0.2380 \\
\mathrm{P}_{2} & -0.3542 & 0.0000 & 0.1683 & 0.1065 & 0.1844 \\
\mathrm{P}_{3} & -0.5314 & -0.3961 & 0.0000 & -0.3961 & -0.3068 \\
\mathrm{P}_{4} & -0.3542 & -0.2505 & 0.1683 & 0.0000 & 0.2129 \\
\mathrm{P}_{5} & -0.5601 & -0.4319 & 0.1304 & -0.5010 & 0.0000
\end{array}\right] \\
& Z_{3}=\left[\begin{array}{lllrrr} 
& \Gamma_{1} & \Gamma_{2} & \multicolumn{1}{l}{\Gamma_{3}} & \multicolumn{1}{l}{\Gamma_{4}} & \multicolumn{1}{c}{\Gamma_{5}} \\
\mathrm{P}_{1} & 0.0000 & -0.3308 & -0.4051 & -0.2864 & -0.3308 \\
\mathrm{P}_{2} & 0.3225 & 0.0000 & -0.2339 & 0.2793 & 0.0000 \\
\mathrm{P}_{3} & 0.3950 & 0.2280 & 0.0000 & 0.3225 & 0.2280 \\
\mathrm{P}_{4} & 0.2793 & -0.2864 & -0.3308 & 0.0000 & -0.2864 \\
\mathrm{P}_{5} & 0.3225 & 0.0000 & -0.2339 & 0.2793 & 0.0000
\end{array}\right] \\
& Z_{4}=\left[\begin{array}{rrrrlr} 
& \multicolumn{1}{l}{\Gamma_{1}} & \multicolumn{1}{l}{\Gamma_{2}} & \multicolumn{1}{l}{\Gamma_{3}} & \multicolumn{1}{l}{\Gamma_{4}} & \multicolumn{1}{l}{\Gamma_{5}} \\
\mathrm{P}_{1} & 0.0000 & -0.3351 & 0.2105 & 0.1592 & -0.3746 \\
\mathrm{P}_{2} & 0.1592 & 0.0000 & 0.1592 & 0.2105 & 0.1779 \\
\mathrm{P}_{3} & -0.4433 & -0.3351 & 0.0000 & 0.2105 & -0.4423 \\
\mathrm{P}_{4} & -0.3351 & -0.4433 & -0.4433 & 0.0000 & -0.5026 \\
\mathrm{P}_{5} & 0.1779 & 0.2105 & 0.2105 & 0.2387 & 0.0000
\end{array}\right] \\
& Z_{5}=\left[\begin{array}{rrrrrr} 
& \Gamma_{1} & \multicolumn{1}{l}{\Gamma_{2}} & \multicolumn{1}{l}{\Gamma_{3}} & \Gamma_{4} & \multicolumn{1}{c}{\Gamma_{5}} \\
\mathrm{P}_{1} & 0.0000 & -0.4619 & 0.1155 & 0.1527 & 0.1732 \\
\mathrm{P}_{2} & 0.1155 & 0.0000 & 0.1414 & 0.1527 & 0.1527 \\
\mathrm{P}_{3} & -0.4619 & -0.5657 & 0.0000 & 0.1000 & 0.0000 \\
\mathrm{P}_{4} & -0.6110 & -0.6110 & -0.4000 & 0.0000 & -0.4619 \\
\mathrm{P}_{5} & -0.6928 & -0.6110 & 0.0000 & 0.1155 & 0.0000
\end{array}\right]
\end{aligned}
$$

Step5. By using (5.9), We can determine the overall dominance of each alternative $\mathrm{P}_{i}$ w.r.t the alternatives $\mathrm{P}_{t_{1}}$ and $\widehat{D}$ shows the overall dominance matrix :

$$
\widehat{D}=\left[\begin{array}{rrrrll} 
& \multicolumn{1}{c}{\Gamma_{1}} & \Gamma_{2} & \multicolumn{1}{l}{\Gamma_{3}} & \multicolumn{1}{c}{\Gamma_{4}} & \multicolumn{1}{c}{\Gamma_{5}} \\
\mathrm{P}_{1} & 0.2069 & 0.7648 & -1.3531 & -0.3400 & -0.0205 \\
\mathrm{P}_{2} & -1.7827 & 0.1050 & 0.3679 & 0.7068 & 0.5623 \\
\mathrm{P}_{3} & -1.4902 & -1.6304 & 1.1735 & -1.0112 & -0.9276 \\
\mathrm{P}_{4} & 0.7453 & -0.2235 & -0.6243 & -1.7243 & -2.0839 \\
\mathrm{P}_{5} & -0.5737 & -1.3626 & 0.3679 & 0.2525 & -1.1883
\end{array}\right]
$$

Step 6. With the help of (5.11) and (5.12), we calculate the positive and the negative solution denoted by $\widehat{D}^{\mathrm{p}}$ and $\widehat{D}^{n}$ respectively :

$$
\begin{gathered}
\widehat{D}^{\mathrm{p}}=\left\{\widehat{D}_{1}^{\mathrm{p}}, \widehat{D}_{2}^{p}, \widehat{D}_{3}^{\mathrm{p}}, \widehat{D}_{4}^{p}, \widehat{D}_{5}^{\mathrm{p}}\right\}=(0.7453,0.7648,1.1735,0.7068,0.5623) \\
\widehat{D}^{n}=\left\{\widehat{D}_{1}^{n}, \widehat{D}_{2}^{n}, \widehat{D}_{3}^{n}, \widehat{D}_{4}^{n}, \widehat{D}_{5}^{n}\right\}=(-1.7827,-1.6304,-1.3531,-1.7243,-2.0839)
\end{gathered}
$$


Steps 7-8. In this step, we calculate $L_{i}$ and $G_{i}$ as below:

$$
\begin{aligned}
& L_{1}=0.5990, L_{2}=0.6040, L_{3}=0.3628, L_{4}=0.5489, L_{5}=0.5087 \\
& G_{1}=0.3449, G_{2}=0.3900, G_{3}=0.1500, G_{4}=0.2756, G_{5}=0.2196
\end{aligned}
$$

Step 9. Determine $M_{i}(i=1,2,3,4,5)$ by using the value of $\xi=0.5$

$$
M_{1}=0.8956, M_{2}=1.000, M_{3}=0.000, M_{4}=0.6475, M_{5}=0.4474
$$

\begin{tabular}{|c|c|c|c|c|c|c|c|}
\hline & $\mathrm{P}_{1}$ & $\mathrm{P}_{2}$ & $\mathrm{P}_{3}$ & $\mathrm{P}_{4}$ & $\mathrm{P}_{5}$ & Ranking & $\begin{array}{l}\text { Compromise } \\
\text { solutions }\end{array}$ \\
\hline$L$ & 0.5990 & 0.604 & 0.3628 & 0.5489 & 0.5087 & $\mathrm{P}_{3}>\mathrm{P}_{5}>\mathrm{P}_{4}>\mathrm{P}_{1}>\mathrm{P}_{2}$ & $\mathrm{P}_{3}$ \\
\hline$G$ & 0.3449 & $\begin{array}{ll} & 0.390 \\
0 & \end{array}$ & 0.1500 & 0.2756 & 0.2196 & $\mathrm{P}_{3}>\mathrm{P}_{5}>\mathrm{P}_{4}>\mathrm{P}_{1}>\mathrm{P}_{2}$ & $\mathrm{P}_{3}, \mathrm{P}_{5}$ \\
\hline$M$ & 0.8956 & $\begin{array}{ll} & 1.000 \\
0 & \end{array}$ & 0.000 & 0.6475 & 0.4474 & $\mathrm{P}_{3}>\mathrm{P}_{5}>\mathrm{P}_{4}>\mathrm{P}_{1}>\mathrm{P}_{2}$ & $\mathrm{P}_{3}, \mathrm{P}_{5}$ \\
\hline
\end{tabular}

Step 10. Ranking and compromised solution of the alternatives by taking the values $L, G$ and $M$ is shown in the Table 4.

\begin{tabular}{|c|c|c|c|c|c|c|c|c|}
\hline & $\xi$ & $\mathrm{P}_{1}$ & $\mathrm{P}_{2}$ & $\mathrm{P}_{3}$ & $\mathrm{P}_{4}$ & $\mathrm{P}_{5}$ & Ranking & se $\begin{array}{c}\text { Compromi } \\
\text { solutions }\end{array}$ \\
\hline$L$ & & $90^{0.59}$ & $40^{0.60}$ & $28^{0.36}$ & 0.5489 & 0.5087 & $\mathrm{P}_{3}>\mathrm{P}_{5}>\mathrm{P}_{4}>\mathrm{P}_{1}>\mathrm{P}_{2}$ & $\mathrm{P}_{3}, \mathrm{P}_{5}$ \\
\hline \multirow[t]{6}{*}{$G$} & & $49^{0.34}$ & $00^{0.39}$ & $00^{0.15}$ & 0.2756 & 0.2196 & $\mathrm{P}_{3}>\mathrm{P}_{5}>\mathrm{P}_{4}>\mathrm{P}_{1}>\mathrm{P}_{2}$ & $\mathrm{P}_{3}$ \\
\hline & 0 & $21^{0.81}$ & $00^{1.00}$ & $0_{00} 0.00$ & 0.5233 & 0.2900 & $\mathrm{P}_{3}>\mathrm{P}_{5}>\mathrm{P}_{4}>\mathrm{P}_{1}>\mathrm{P}_{2}$ & $\mathrm{P}_{3}$ \\
\hline & 0.1 & $88^{0.82}$ & $00^{1.00}$ & $00^{0.00}$ & 0.5481 & 0.3215 & $\mathrm{P}_{3}>\mathrm{P}_{5}>\mathrm{P}_{4}>\mathrm{P}_{1}>\mathrm{P}_{2}$ & $\mathrm{P}_{3}$ \\
\hline & 0.2 & $55^{0.84}$ & $00^{1.00}$ & $0_{00} 0.00$ & 0.5730 & 0.3530 & $\mathrm{P}_{3}>\mathrm{P}_{5}>\mathrm{P}_{4}>\mathrm{P}_{1}>\mathrm{P}_{2}$ & $\mathrm{P}_{3}$ \\
\hline & 0.3 & $22^{0.86}$ & $00^{1.00}$ & $0_{00} 0.00$ & 0.5978 & 0.3845 & $\mathrm{P}_{3}>\mathrm{P}_{5}>\mathrm{P}_{4}>\mathrm{P}_{1}>\mathrm{P}_{2}$ & $\mathrm{P}_{3}$ \\
\hline & 0.4 & $89^{0.87}$ & $00^{1.00}$ & $00^{0.00}$ & 0.6226 & 0.4160 & $\mathrm{P}_{3}>\mathrm{P}_{5}>\mathrm{P}_{4}>\mathrm{P}_{1}>\mathrm{P}_{2}$ & $\mathrm{P}_{3}$ \\
\hline \multirow[t]{5}{*}{$M(v)$} & 0.5 & $57^{0.89}$ & $00^{1.00}$ & $0_{00} 0.00$ & 0.6474 & 0.4474 & $\mathrm{P}_{3}>\mathrm{P}_{5}>\mathrm{P}_{4}>\mathrm{P}_{1}>\mathrm{P}_{2}$ & $\mathrm{P}_{3}, \mathrm{P}_{5}$ \\
\hline & 0.6 & $24^{0.91}$ & $00^{1.00}$ & $00^{0.00}$ & 0.6723 & 0.4789 & $\mathrm{P}_{3}>\mathrm{P}_{5}>\mathrm{P}_{4}>\mathrm{P}_{1}>\mathrm{P}_{2}$ & $\mathrm{P}_{3}$ \\
\hline & 0.7 & $91^{0.92}$ & $00^{1.00}$ & $00^{0.00}$ & 0.6971 & 0.5104 & $\mathrm{P}_{3}>\mathrm{P}_{5}>\mathrm{P}_{4}>\mathrm{P}_{1}>\mathrm{P}_{2}$ & $\mathrm{P}_{3}$ \\
\hline & 0.8 & $588^{0.94}$ & $00^{1.00}$ & $0_{00} 0.00$ & 0.7219 & 0.5419 & $\mathrm{P}_{3}>\mathrm{P}_{5}>\mathrm{P}_{4}>\mathrm{P}_{1}>\mathrm{P}_{2}$ & $\mathrm{P}_{3}$ \\
\hline & 0.9 & 0.96 & 1.00 & 0.00 & 0.7467 & 0.5734 & $\mathrm{P}_{3}>\mathrm{P}_{5}>\mathrm{P}_{4}>\mathrm{P}_{1}>\mathrm{P}_{2}$ & $\mathrm{P}_{3}$ \\
\hline
\end{tabular}

Table 3: The ranking and comromised solution 


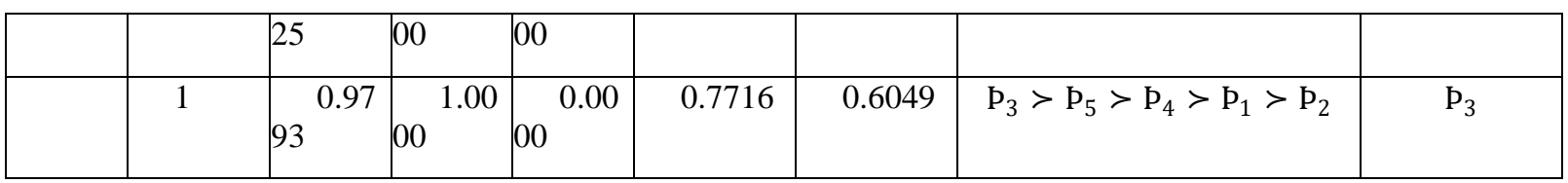

Table 4: Ranking and compromised solution obtained with the change of weight $\xi$

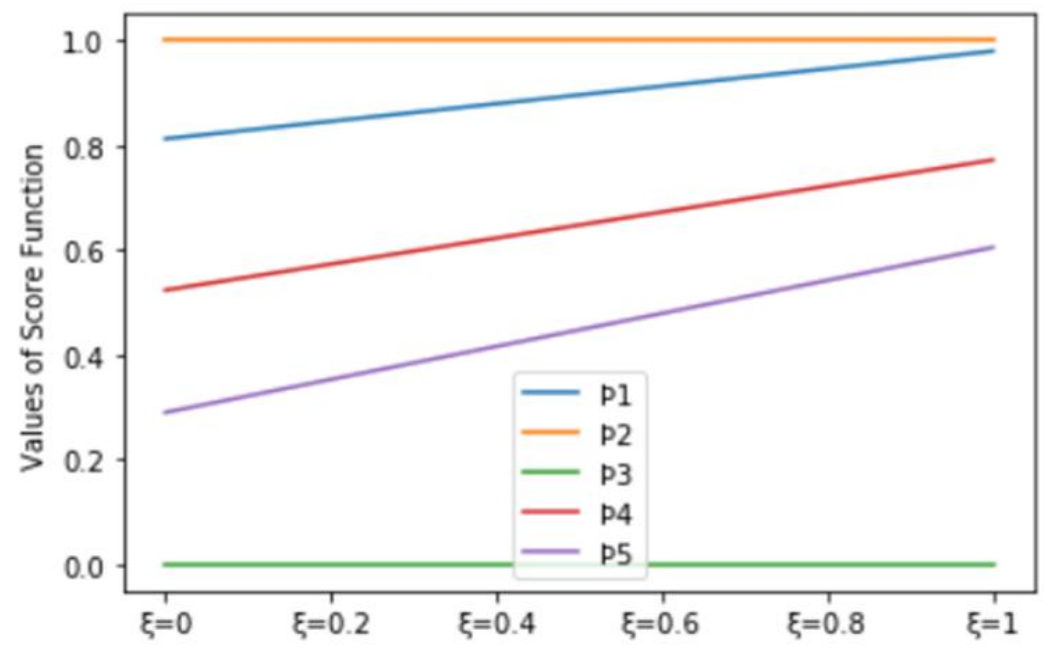

Figure 1: The line graph of the alternatives under the $L, G$ and $M(\xi)$ at different values
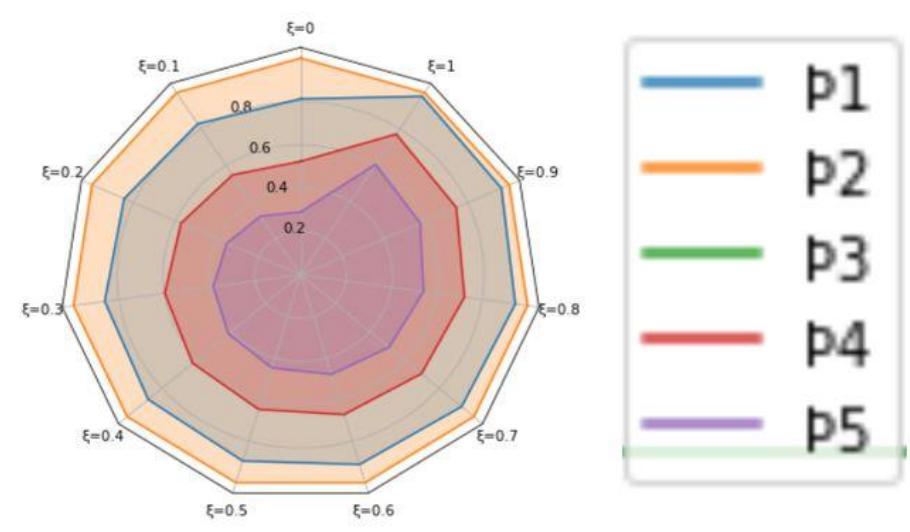

Figure 2: The sensitivity analysis of the alternatives under the $L, G$ and $M(\xi)$ at different values of $\xi$

$\xi$ plays a vital role for to find the values of $\mathrm{M}$ and also in final result analysis of proposed approach, see (5.15), which represents the weight of the approach of the utmost group utility. In fact, when $\xi=0, M$ only depicts the least of the individual regret for the opponent $G_{i}$. If $\xi=1, M$ becomes the utmost group utility of the majority $L_{i}$. In general, we discuss the influence of $\xi$ to the value of $M$. The results are appeared in Table 4. From Table 4, we can say that those distribution graphics have the same distribution when the weight $\xi \leq 0.5$ or $\xi \geq 0.5$ and the values of $M_{i}$ values of five possible projects have the same change rate as the weight $\xi$ increases. So, the best project is different as the weight $\xi$ increases. With the results in table 4 and visualized results in Fig. 1 and Fig. 2, the schemes provided the best choice $\mathrm{P}_{3}$ or $\left(\mathrm{P}_{3}, \mathrm{P}_{5}\right)$. Step 11. Results in Table 4 demonstrate that alternatives $P_{3}$ and $P_{5}$ places at the first two positions in the ranking. However, by using the condition X1, $M\left(\mathrm{p}^{(3)}\right)-M\left(\mathrm{p}^{(5)}\right)=0.000-0.4474=-0.4474<\frac{1}{5-1}=0.25$. Which shows that the condition $\mathbf{X} 1$ is not satified. Therefore, we look for the compromise solution given below :

$$
M\left(\mathrm{P}^{(5)}\right)-M\left(\mathrm{P}^{(3)}\right)=0.4474-0.000=0.4474<\frac{1}{5-1}=0.25 \text {. Thus, } \mathrm{P}_{3} \text { and } \mathrm{P}_{5} \text { are our compromised }
$$
solutions. 


\subsection{Approch 2: For Completely Unknown Weights}

In this subsection, when the criteria weights are completely unkown then we solve the same example :

1. By using (5.5), we will determine the values of criteria weights as follows:

$$
w_{1}=0.199, w_{2}=0.201, w_{3}=0.190, w_{4}=0.207, w_{5}=0.203
$$

2. Ranking and compromised solution the alternatives, by taking the values $L, G$ and $M$ is shown in the Table 5 .

\begin{tabular}{|c|c|c|c|c|c|c|c|}
\hline & $\mathrm{P}_{1}$ & $\mathrm{P}_{2}$ & $\mathrm{P}_{3}$ & $\mathrm{P}_{4}$ & $\mathrm{P}_{5}$ & Ranking & $\begin{array}{c}\text { Compromis } \\
\text { solutions }\end{array}$ \\
\hline$L$ & 0.5674 & 0.6104 & 0.3523 & 0.5583 & 0.4824 & $\mathrm{P}_{3}>\mathrm{P}_{5}>\mathrm{P}_{4}>\mathrm{P}_{1}>\mathrm{P}_{2}$ & $\mathrm{P}_{3}$ \\
\hline$G$ & 0.3223 & 0.3804 & 0.1427 & 0.2652 & 0.2304 & $\mathrm{P}_{3}>\mathrm{P}_{5}>\mathrm{P}_{4}>\mathrm{P}_{1}>\mathrm{P}_{2}$ & $\mathrm{P}_{3}, \mathrm{P}_{5}$ \\
\hline $\boldsymbol{M}$ & $\mathbf{0 . 8 7 9 4}$ & $\mathbf{1 . 0 0 0 0}$ & $\mathbf{0 . 0 0 0}$ & $\mathbf{0 . 6 3 5 2}$ & $\mathbf{0 . 4 9 5 6}$ & $\mathrm{P}_{\mathbf{3}}>\mathrm{P}_{\mathbf{5}}>\mathrm{P}_{\mathbf{4}}>\mathrm{P}_{\mathbf{1}}>\mathrm{P}_{\mathbf{2}}$ & $\mathrm{P}_{\mathbf{3}}, \mathrm{P}_{\mathbf{5}}$ \\
\hline
\end{tabular}

Table 5: The ranking and compromised solution

3. It is clear from the table 5, the alternatives $\mathrm{P}_{3}$ and $\mathrm{P}_{5}$ places at the first two positions in the grading list of $M_{i}$. For condition X1, $M\left(\mathrm{p}^{(3)}\right)-M\left(\mathrm{p}^{(5)}\right)=0.000-0.4956=-0.4956<\frac{1}{5-1}=0.25$, that shows that Condition X1 is not satisfoed, therefore we look for the compromised solution which is given below:

$Q\left(\mathrm{P}^{(5)}\right)-Q\left(\mathrm{P}^{(3)}\right)=0.4956-0.0000=0.4956>\frac{1}{5-1}=0.25$. Then, $\mathrm{P}_{3}$ and $\mathrm{P}_{5}$ are the compromised solutions.

Therefore, the compromised solutions remains the same by both methods.

\section{Comparative analysis}

To verify the effectiveness of our proposed entropy we compare it with the method proposed by (Wei [5]; Amalendu et al. [17];Chunxin and Zhang [21];Nei [10]) and computed the same example with the same weight information as shown in table 6.

\begin{tabular}{|c|c|c|}
\hline Method Proposed by & Methods & Ranking \\
\hline Wei [5] & Cross entropy & $\mathrm{P}_{3}>\mathrm{P}_{5}>\mathrm{P}_{1}=\mathrm{P}_{4}>\mathrm{P}_{2}$ \\
\hline Amalendu et al. [17] & $\begin{array}{c}\text { New ranking } \\
\text { method }\end{array}$ & $\mathrm{P}_{3}>\mathrm{P}_{5}>\mathrm{P}_{1}>\mathrm{P}_{4}>\mathrm{P}_{2}$ \\
\hline Chunxin and Zhang [21] & Score function & $\mathrm{P}_{3}>\mathrm{P}_{1}>\mathrm{P}_{4}>\mathrm{P}_{2}>\mathrm{P}_{5}$ \\
\hline Nei, [10] & Comparison rule & $\mathrm{P}_{3}=\mathrm{P}_{5}>\mathrm{P}_{2}>\mathrm{P}_{1}>\mathrm{P}_{4}$ \\
\hline New method & TODIM-VIKOR & $\mathrm{P}_{\mathbf{3}}>\mathrm{P}_{\mathbf{5}}>\mathrm{P}_{\mathbf{4}}>\mathrm{P}_{\mathbf{1}}>\mathrm{P}_{2}$ \\
\hline
\end{tabular}

Table 6: Comparison of Ranking results

The ranking of alternatives so obtained is given by : $\mathrm{P}_{3}>\mathrm{P}_{5}>\mathrm{P}_{4}>\mathrm{P}_{1}>\mathrm{P}_{2}$, thus $\mathrm{P}_{3}$ as the most suitable alternative. In our proposed method, $\mathrm{P}_{3}$ is best choice, but ranking order does not matter for other alternatives. In the former methods, the weights criteria are assumed by experts or determined by aggregation operators, which can be unreasonable to be attained practically. Compared with the existing methods, the latter (proposed approach) has some valuable advantages as follows:

(a)

In a complex decision making context, using PFNs that involve various types of evaluating results to represent experts view is a good choice.

The entropy approach is used for the calculation of the criterion weight and this approach is more reasonable and flexible.

(c)

The advantages of entropy information, experts behaviours , group utility and minimum individual regret are fully used

\section{Conclusions}

In this paper, we conclude that a new fuzzy information has been successfully introduced and validated it in light of newly proposed framework for PFSs which is an extension of IFS. Realizing the vital role of criteria 
weights in MCDM problems, the proposed MCDM problem has dicussed by applying two different approaches that is partially known and completely unkown criteria weight. PFSs are appropriate in describing and addressing the uncertainty and vagueness information measure occurring in MCDM problems. Additionally, the operating of proposed MADM method is throughly explained with the help of numerical example based on the concept of PF VIKOR-TODIM supported opinion polls for predicting the output of elections. To check the viability and applicability of the proposed MCDM method, we compare the resulting output with the existing MCDM in literatures. With better practical decision making value, the eminent achivements of the present research can forward an effective and reliable scientific approach for solving the multi criteria picture fuzzy decision making problem. The proposed MCDM method is applied to various complicated problems like site choice, venture establishment, health department, insurance sector where it helps to determine the risk factor, to establish new venture and so on.

\section{References}

Zadeh LA (1965) Fuzzy sets, Inform Control 8:338-353

Zhang XH,Pei DW, Dai JH (2013) Fuzzy Mathematics and Rough Set

theory; Tsinghua University Press :Beijing, China.

Atanassov KT (1986) Intuitionistic fuzzy sets,Fuzzy Set Syst.1986; 20:87-96.

Torra V.(2010) Hesitant fuzzy sets . Int J Intell Syst 25:529-539 .

Wei GW (2016) Picture fuzzy cross-entropy for multiple attribute decision making problems.Journal of Business Economics and Management. 17(4): 491- 502.

Wang CZ, Shao MW, He Q (2016) Feature subset selection based on fuzzy sets.Knowl-Based Syst 111:173-179.

Arya V, Kumar S (2020a) Multi-criteria decision making problem for evaluating ERP system using entropy weighting approach and q-rung orthopair fuzzy TODIM. Granular Computing. https://doi.org/10.1007/s41066-020-00242-2.

Cuong BC (2013)Picture Fuzzy Sets-First results,Part 1,seminar, Neuro-Fuzzy Systems with Applications';Preprint 03/2013 and Preprint 04/2013;Institute of mathematics:Hanoi,Vietnam.

Zadeh LA (1975)The concept of linguistic variable and its application to approximate reasoning-I Inform Sci 8:199-249

Nie RX, Wang JQ, Li LA (2017) shareholder voting method for proxy advisory firm selection based on 2-tuple linguistic picture preference relation. Applied Soft Comput 60:520-539.

Peng X, Dai (2017) Algorithm for picture fuzzy multiple attribute decision making based on new distance measure, Int J Uncertain Quant 7: 177-187.

Son H (2016) Generalized Picture Distance Measure and Applications to Picture Fuzzy Clustering. Applied Soft Comput 46:284-295.

Yu PL (1973)A class of solutions for group decision problems. Manage Sci 19 (8):936-946 .

Hung WL., Yang MS.( 2006) Fuzzy entropy on intuitionistic fuzzy sets. Int. J. Intell. Syst. 21(4) 443-451.

Cuong BC (2014) Picture fuzzy sets, Journal of Computer Science and Cybernetics 30(4):409-420.

Cuong BC, Kreinovich V (2013)Picture Fuzzy Sets-a new concept for computational intelligence problems, Third World Congress on Information and Communication Technologies.809.

Amalendu SI,Das S,Kar S (2019)An approach to rank picture fuzzy numbers for decision making problems. Decision making. https://doi.org/10.31181/dmame1902049s .

[18] Verlag, Berlin.

Fan ZP, Zhang X, Chen FD, Liu Y (2013)Extended TODIM method for hybrid multiple attribute decision making problems. Knowl-Based Syst 42:40-48 .

Arya V, Kumar S (2020c) Fuzzy entropy measure with an applications in decision making under bipolar fuzzy environment based on TOPSIS method. Int J Inf Manag Sci 31(2):99-121.

Chunxin Bo, Zhang X (2017)New Operations on Interval-Valued Picture Fuzzy Set, Interval-Valued Picture Fuzzy Soft Set and their Applications 9(11):268.

Lourenzutti R, Krohling RA (2013) A study of TODIM in a intuitionistic fuzzy and random environment Exp Syst Appl 40: 6459-6468.

Krohling RA, Pacheco AGC, Siviero ALT (2013) IF-TODIM: An intuitionistic fuzzy TODIM to multicriteria decision making. Knowl- Based System 53: 142-146 .

Konwar N, Debnath P (2017)Continuity and Banach contraction principle in intuitionistic fuzzy n normed linear spaces, J Intell and Fuzzy Syst 33(4) 2363-2373.

Wei GW, Alsaadi FE, Hayat T, Alsaedi A (2018) Bipolar fuzzy Hamacher aggregation operators in multiple attribute decision making. Int J Fuzzy Syst 20(1):1-12.

Opricovic S (1988)Multicriteria optimization of civil engineering sytem, Faculty of Civil Engineering, Belgrade, Serbia 
Kumar S , Kumar S (2020) A generelization of Gini Simpson Index under fuzzy Environment, Advances in Mathematics : Scientific Jounal 9, no.8, 5443-5454. 20(1):1-12.

Wang C, Zhou X, Tu H, Tao S (2017)Some Geometric Aggregation Operators based On Picture Fuzzy Setsand Their Application in Multiple Attribute Decision Making.Italian J of Pure and Applied Mathematics 37:477492.

De Luca A,Termini S (1972) A definition of non-probabilistic entropy in setting of fuzzy set theory. Inform control20:301-312.

Szmidt E,Kacprzyk J (2000) Distances between intuitionistic fuzzy sets.Fuzzy Set Sci 67(3):204-228. 